\title{
Prosthetic rehabilitation of hemi-mandibulectomy Patient with a definitive prosthesis - a case report
}

\author{
Doddy Lokanathan Balaji ${ }^{1}$, P. Sesha Reddy ${ }^{2, *}$ \\ ${ }^{1}$ Postgraduate student, Government Dental College and Hospital, Kadapa, Andhra Pradesh, India. \\ 2Professor, Government Dental College and Hospital, Kadapa, Andhra Pradesh, India.
}

\section{N F O R M A T I O N}

\section{Article History}

Received 30 September 2019

Received revised

14 October 2019

Accepted 15 October 2019

Available online

15 November 2019

\section{K E Y W O R D S}

Hemimandibulectomy

Cast partial denture prosthesis

Resected ameloblastoma

Fibular bone graft

\section{A B S T R A C T}

"Often the loss reveals the value of things". Surgical removal of odontogenic tumours in the posterior region of mandible leads to discontinuity of mandible. Prosthetic rehabilitation is a successful treatment modality to reconstructive surgeries. Mandibular resection leads to deviated mandibular movements, disfigurement, facial profile asymmetry, dysphagia, impaired speech and, deviation of the mandible towards the surgical site. Treatment modality for oral rehabilitation of patients with mandibular resection includes maxilla-mandibular fixation, implant-supported prosthesis, removable mandibular guide flange prosthesis, and twin occlusion prosthesis. One of the treatment options for the mandibular defect is Cast partial denture prosthesis, whereas surgical reconstruction by implants and grafts is always not feasible in every patient. This clinical report describes rehabilitation of Cantor and Curtis class III mandibular defect using cast partial denture prosthesis following hemi-mandibulectomy.

\section{Introduction}

Ameloblastoma is a slow-growing benign tumour of the jaw. The most common site for ameloblastoma is mandibular unerupted third molar region. Ameloblastoma of the lower jaw is an aggressive benign tumour of epithelial origin. It is the second most common odontogenic tumour. There are three forms of ameloblastoma, namely peripheral, unicystic, and multicystic tumour. Multicystic ameloblastoma is commonly seen among all and represents $90 \%$ of cases [1,2]. Diagnosis mainly from tissue biopsy and characteristic finding on X-ray OPG assist in differentiating between types of ameloblastoma. The challenges in the management of this tumour are to provide complete excision and reconstruction of the bony defect to restore cosmetic and functional outcome to the patient. Restoration of facial form, function, and aesthetics in a patient who has undergone hemi mandibulectomy is a valuable service rendered by a prosthodontist [3,4]. A classification of mandibular defects has been described by cantor and Curtis. Although the classification system is suggested primarily for edentulous patients, it is also applicable to partially edentulous patients. This defect classified based on remaining structures $[5,6,7]$.

Correspondence: ${ }^{*}$ Corresponding author Email Address: $\underline{\text { drpseshareddy@gmail.com }}$

How to cite this article: Balaji DL, Sesha Reddy P. Prosthetic rehabilitation of hemi-mandibulectomy Patient with a definitive Prosthesis - a case report. Int J Dent Mater 2019;1(2): 68-71. 


\section{Cantor and Curtis classification (figure 1) (1971) $[5,6,7]$}

Class 1: Radical alveolectomy with preservation of mandibular continuity.

Class 2: Lateral resection of mandible distal to cuspid. Class 3: Lateral resection of mandible involves loss up to the midline.

Class 4: Lateral bone graft surgical reconstruction.

Class 5: Anterior bone graft surgical reconstruction.

Class 6: Resection of the anterior portion of the mandible without reconstructive surgery.

\section{Case report}

An 18 years old female patient reported to the Department of Prosthodontics (Government Dental College and Hospital, Kadapa) with a chief complaint of missing teeth in lower right teeth region of the jaw. Past dental history revealed that she was diagnosed as Ameloblastoma in the right posterior mandibular region (figure 2). The patient underwent excision of the lesion followed by the resection of a portion of the mandible and placement of avascular fibular bone graft with titanium reconstructive plate was placed to restore the resected defects (figure 3). On extraoral examination showed symmetrical face, concave profile. On intraoral examination revealed that knife-edge ridge with shallow sulcus depth and partially edentulous fourth quadrant teeth (figures 4 and 5). Based on the clinical situation, a removable cast partial denture was planned.

\section{Clinical procedure}

The patient was evaluated for the fabrication of a cast partial removable partial denture prosthesis. Primary impression was made, and the diagnostic cast was prepared. Surveying was done to assess the number of undercuts on primary abutments and to assess the Path of insertion and removal of the prosthesis. The Removable partial denture includes occlusal rest seats on the $34,35,36$ teeth region. The occlusal rest seats were prepared on the distal marginal ridge and the mesial marginal ridge on the first and second premolars and the first molar respectively. The Major connector was a lingual plate, and an I-Bar placed on the 31 teeth and Minor connector supporting the occlusal rim was meshwork type which will be replaced by denture bases with teeth at a later stage. The Direct retainer planned was embrasure clasp on the second premolar and molar teeth whereas simple circlet clasp in the first premolar region. After mouth preparation, the secondary impression was made using Poly vinyl

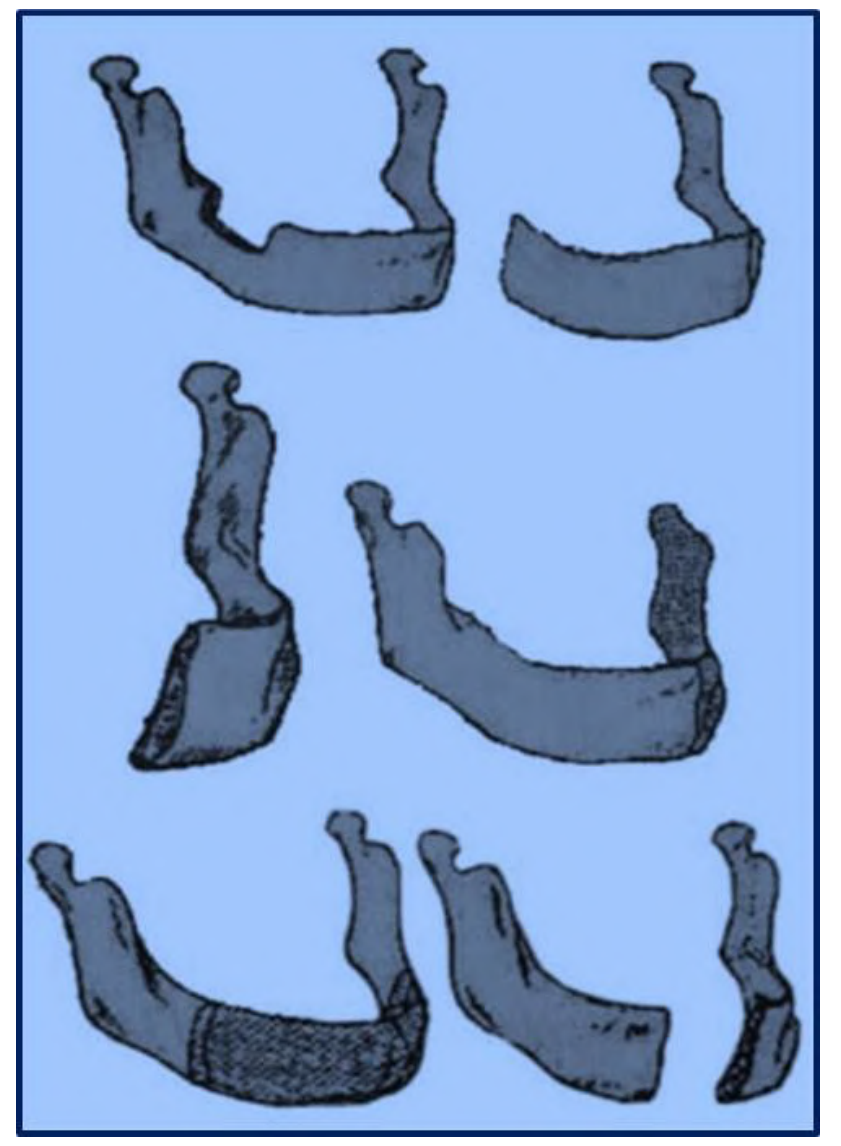

Figure 1. Cantor and Curtis classification of mandibular defects

siloxane putty (Aquasil -Addition Silicone) and Light bodied (Aquasil -Addition Silicone) elastomeric impression material using putty wash technique (figure $6)$. The master cast was poured using type IV die stone. On the master cast, surveying was done (figure 7).

Planned Block out procedure was carried out and cast duplicated using agar (Castogel, BEGO). Ethyl silicate bonded investment material (Wirovest) was used to obtain a refractory cast over which wax pattern was adapted. The casting was done to obtain a metal framework, which was then tried in the patient mouth for fit, over which modelling wax was adapted to get an occlusal rim. The maxillary occlusal plane was transferred to articulator using face-bow and bite registration was recorded, and articulation was done in a semiadjustable articular (figures 8 and 9).

After teeth arrangement, the wax trial was checked in the patient mouth (figures 10 and 11). After try-in procedure processing of denture was done using an injection moulding technique. Finally, trimmed and polished cast partial was inserted in the patient mouth (figures 12). After regular recall, patient was satisfactory with the prosthesis and maintained good oral hygiene (figures 13,14 and 15). 

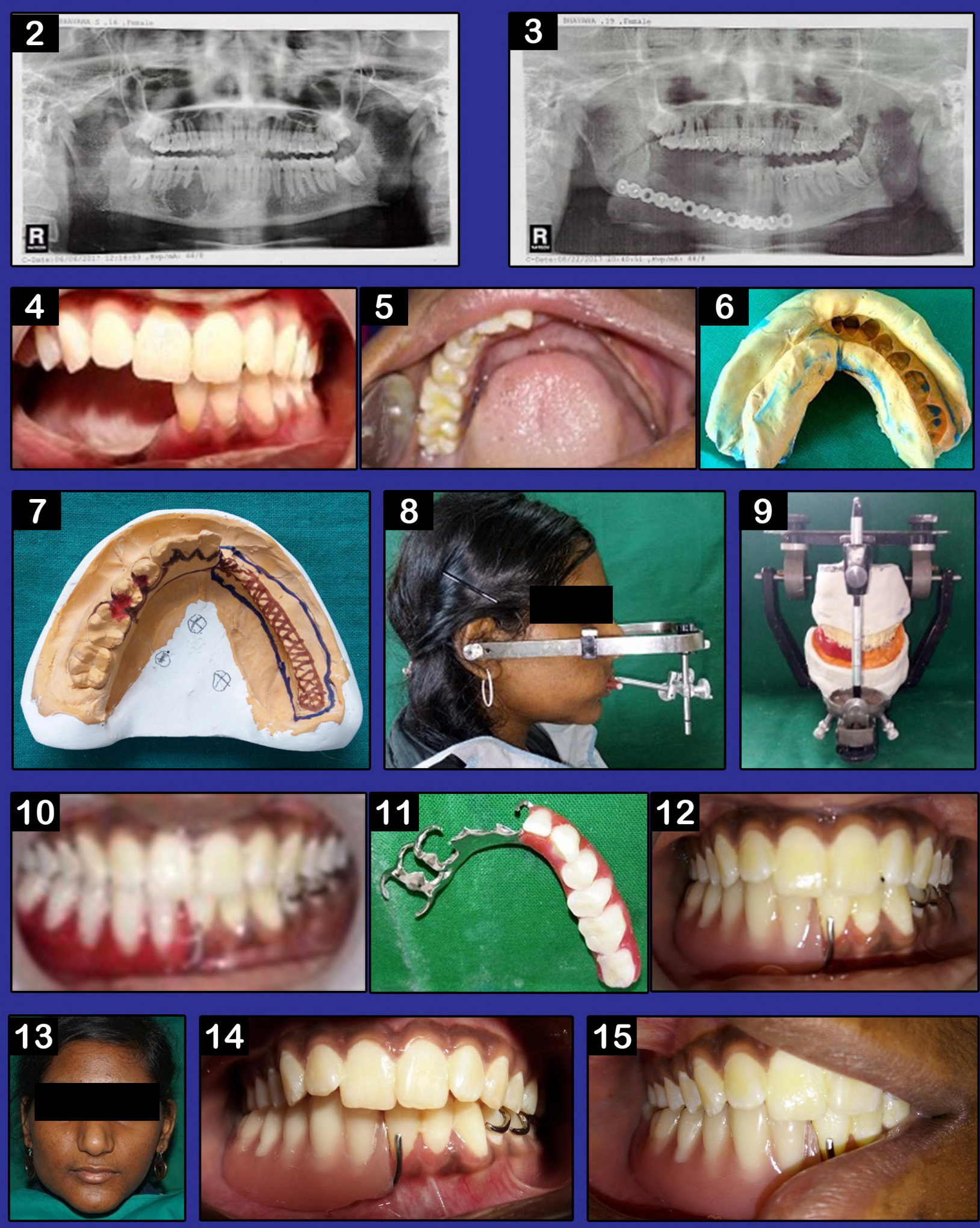

Figures: 2. Pre-operative ameloblastoma 3. Post-operative resected ameloblastoma 4 \& 5. Intraoral Views 6 . Secondary impression (primary putty material with secondary light bodies material)7. Surveying master cast 8 . Face-bow transfer 9. Articulation 10.Try-in 11. Trial wax Cast partial denture 12. Insertion view 13. Follow-up - Extra-oral view $14 \& 15$. Follow-up - Intra oral views 


\section{Discussion}

Loss of mandibular continuity results in deviation of remaining mandibular segment toward the resected side primarily because of loss of tissue involved in the surgical resection.it also causes rotation of mandibular occlusal plane inferiorly on the defect side. The pull of suprahyoid muscles on the residual mandibular resected part causes inferior displacement and rotation around the fulcrum of the remaining condyle, thus giving the tendency to an open bite anteriorly $[8,9]$. Greater the loss of tissue more will be the deviation of the mandible to the resected side, thus compromising the prognosis of the treatment. In this case, titanium reconstructive plates create peripheral boundaries for the floor of the oral cavity and restore the form of face to a certain extent. Inadequate retention, stability, and support are common prosthodontics treatment problems for patients who underwent hemi mandibulectomy[9,10].

Cantor and Curtis classification system for mandibulectomy describes the basic principles to be strictly adhered to in designing a removable cast framework for partially edentulous patients. Usually, a quadrilateral or tripodal design is favoured over a linear design because this allows a more favourable distribution of forces for enhancing the support, stabilization, and retention of the prosthesis $[11,12,13]$. In dentulous patients, the number and distribution of remaining teeth determine the primary retention, support, and stability of the prosthesis $[14,15]$. This report described Cast partial denture prosthesis to restore the form and shape of the missing structure such as alveolar bone with teeth and gives the necessary labial support to the lower lip. The rest seat was prepared to engage the occlusal rest from which embrasure clasps arise and provide the required retention to the prosthesis $[14,15]$.

After regular recall the patient, clinical examination showed that the patient was maintaining satisfactory oral hygiene. The patient was able to chew well and was extremely satisfied with the results.

\section{Conclusion}

A proper diagnosis and a well - designed treatment plan will result in pleasant outcomes. Rehabilitation with cast partial denture prosthesis for reconstructing the mandibular defects leads to improved function, cosmetics, and superior quality of life in appropriately selected patients. Maxillofacial prosthesis is important not only in rehabilitation and aesthetics, but also it improves patient confidence and resocialization in society.

\section{References}

1. Beumer J, CurtisT, Marunick MT. Maxillofacial Rehabilitation: Prosthodontic and surgical consideration. Ishiyaku Euro America, St. Louis, OK.1996, 184-188

2. Beumer J, Curtis T, Firtell D editors. Maxillofacial rehabilitation. St. Louis: Mosby. 1979, 90-169.

3. Schneider RL, Taylor TO. Mandibular resection guidance prosthesis: A literature review. J Prosthet Dent 1986;55:84-6.

4. Swoope CC.Prosthetic management of resected edentulous mandible. J Prosthet Dent. 1969;21 (2):197-202.

5. Cantor R, Curtis TA. Prosthetic management of edentulous mandibulectomy patients: part II, Clinical procedures. J Prosthet Dent 1971; 25:546-55.

6. Desjardi ns RP. Occlusal considerations for the partial mandibulectomy patients. J Prosthet Dent 1979; 41:308-15.

7. Kenneth FB. Complete denture treatment in patients with the resected mandible. JProsthet Dent 1969; 21:443-7.

8. Sahin N, Hekimoglu C, Aslan Y. The fabrication of cast metal guidance flange prosthesis for a patient with segmental mandibulectomy: a clinical report. J Prosthet Dent 2005; 93:217.

9. Rosenthal LE. The edentulous patient with jaw defects. D Clin N Am 1994; 8:773-779.

10. Cantor, R., Curtis, T.A., Shipp, T., Beumer, J., and Vogel, B.S. Maxillary speech prostheses for mandibular survival defects, J Prosthet Dent. 1969; 22: 253-260.

11. Cantor, R., Curtis T.A.. Prosthetic Management of Edentulous Mandibulectomy patients. Part I. Anatomic, Physiologic, and Psychologic considerations, J Prosthet Dent. 1971; 25:446-457.

12. Robinson, J.E., Rubright, W.C.: Use of a Guide Plane for Maintaining the Residual Fragment in Partial or Hemi mandibulectomy, J Prosthet Dent. 1964; 14:992-999.

13. Ashlesha Subhash Marathe, Prasad Shankar rao Kshisagar. A systematic approach in rehabilitation of hemimandibulectomy: A case report. J Indian Prosthodont Soc 2016;16:208.

14. Ferreira AN, Aras M, Chitre V, Mascarenhas K. Prosthetic Rehabilitation of a Patient with Ameloblastoma using an Unconventional Cast Partial Denture. Dentistry2018; 8:482.

15. Narendra.R, P. Seshareddy, Sashideepth Reddy, S. Arunakumari. Prosthodontic Rehabilitation of Cantor and Curtis Class III Mandibular Defect Using Cast Partial Denture: A Case Report. J Pharm Sci Res. 2016; 8(6):461-463. 